\title{
Nódulos plantares dolorosos tras ejercicio intenso en paciente pediátrica: un reto diagnóstico
}

\author{
Painful nodules on the soles in a pediatric patient: a diagnostic challenge \\ Javier Sánchez-Bernala ${ }^{\mathrm{a}}$, Isabel Zárate-Tejero ${ }^{\mathrm{b}}$, Pilar Collado-Hernández ${ }^{\mathrm{b}}$, \\ Mariano Ara-Martín ${ }^{a}$, Lucía Prieto-Torres ${ }^{\mathrm{a}}$
}

aDepartamento de Dermatología, Hospital Clínico Universitario Lozano Blesa de Zaragoza, España

bDepartamento de Pediatría, Hospital Clínico Universitario Lozano Blesa de Zaragoza, España

Recibido: 3 de septiembre de 2019; Aceptado: 19 de noviembre de 2019

¿Qué se sabe del tema que trata este estudio?

Nódulos plantares dolorosos corresponde a una patología de etiología desconocida, con características clínicas y patológicas bien definidas.
¿Qué aporta este estudio a lo ya conocido?

Se presenta un caso clínico muy ilustrativo de esta patología, probablemente subdiagnosticada, que ayuda al conocimiento de esta entidad, facilitando su manejo y evitando errores diagnósticos que puedan llevar a tratamientos innecesarios.

\section{Resumen}

Introducción: La Hidradenitis palmoplantar ecrina idiopática (HPPI) es una dermatosis neutrofílica infrecuente, que cursa con nódulos eritematosos dolorosos de comienzo brusco en regiones plantares o palmoplantares, en niños que no tienen otra enfermedad subyacente. Objetivo: Presentar un caso que ilustra las principales características clínicas e histológicas de la HPPI. Caso Clínico: Niña de 11 años evaluada por nódulos eritematovioláceos dolorosos en planta de pie derecho de 48 horas de evolución y fiebre de hasta $38,2^{\circ} \mathrm{C}$, sin antecedentes de interés salvo hiperhidrosis y práctica intensa de ejercicio en los días previos. Ante la sospecha clínica de HPPI se realizó biopsia cutánea, que mostró infiltrado inflamatorio neutrofílico alrededor de glándulas sudoríparas ecrinas y abscesos de neutrófilos, confirmando el diagnóstico. Se indicaron antiinflamatorios no esteroidales orales y reposo, con resolución de las lesiones en 7 días. Conclusiones: Este caso demuestra los aspectos más importantes de la HPPI. Esta entidad en muchos casos es infradiagnosticada, dado que puede confundirse con otras patologías que también cursan con nódulos acrales dolorosos, pero tienen distintas implicaciones patogénicas y terapéuticas. Identificar apropiadamente la HPPI permite evitar la alarma innecesaria, tanto en pacientes y sus padres, como en los propios dermatólogos y pediatras.
Palabras clave:

Hidradenitis;

dermatosis de los pies/ diagnóstico; patología de glándulas ecrinas;

niños

Correspondencia:

Javier Sánchez Bernal

javisanchez_5@hotmail.com 


\section{Abstract}

Introduction: Idiopathic Palmoplantar Eccrine Hidradenitis (IPPH) is a rare neutrophilic dermatosis, with painful erythematous nodules of sudden onset in the plantar or palmoplantar region, in children without other underlying diseases. Objective: To present a case that shows the main clinical and histological characteristics of this entity. Clinical Case: 11-year-old girl with a 48-hours history of painful erythematous-violaceous nodules on the right foot plant associated with fever of up to 38.2 ${ }^{\circ} \mathrm{C}$, with no history of interest except hyperhidrosis and intense exercising on previous days. Given the clinical suspicion of IPPH, a skin biopsy was performed, which showed inflammatory neutrophil infiltration around eccrine sweat glands and neutrophilic abscesses, confirming the diagnosis. Oral NSAIDs and rest were prescribed, with resolution of the lesions in 7 days. Conclusions: This case demonstrates the most important aspects of this entity, in many cases underdiagnosed, since it can be confused with other pathologies that occur with painful acral nodules, but have different pathogenic and therapeutic implications. To properly identify the IPPH allows preventing an unnecessary alarm, both patients and their parents, as in dermatologists and pediatricians themselves.

\section{Keywords:}

Hidradenitis; Foot

Dermatoses/diagnosis; Eccrine Glands/pathology; Child

\section{Introducción}

La hidradenitis palmoplantar ecrina idiopática (HPPI) es una dermatosis neutrofílica infrecuente. Fue descrita por primera vez en 1994 por Stahr et al. ${ }^{1}$ en 6 pacientes sanos, 5 niños y un adulto joven, todos ellos con un cuadro clínico de lesiones dolorosas de afectación plantar y un estudio histológico parecido al descrito en la hidradenitis ecrina neutrofílica (HEN) pero con características diferenciales. Desde entonces se han publicado menos de 50 casos, la mayoría en pacientes sanos de entre 2 y 15 años, con afectación plantar y, en algunas ocasiones, palmar.

Su etiopatogenia no se ha aclarado todavía. Se ha observado una mayor incidencia en los meses calurosos en pacientes con antecedente de ejercicio físico intenso y/o calzado cerrado ${ }^{2,3}$. Algunas teorías defienden que, en pacientes con glándulas inmaduras, un traumatismo mecánico o térmico podría producir la ruptura de las mismas con salida de su contenido a la dermis, activando citocinas inflamatorias con capacidad de atraer polimorfonucleares ${ }^{2,4,5}$. Otra hipótesis plantea que la excesiva sudoración, intrínseca o relacionada con el ejercicio físico intenso, iniciaría toda esta cascada inflamatoria $^{6,7}$. Sin embargo, los estudios de laboratorio no aportan datos específicos al respecto ${ }^{8}$.

Los hallazgos histológicos son característicos y consisten en la presencia de un intenso infiltrado inflamatorio, de predominio neutrofílico, alrededor de las glándulas sudoríparas ecrinas, tanto en la porción del ovillo, en cuyas proximidades pueden formarse también abscesos de neutrófilos, como en el conducto, pudiendo encontrarse además acúmulos de neutrófilos en el interior del mismo de forma inconstante pero bastante específica ${ }^{8}$. No se observan siringometaplasia escamosa ecrina ni signos típicos de vasculitis leucocitoclástica ${ }^{2,8}$.
El objetivo del manuscrito es presentar un caso que ilustra las principales características clínicas e histológicas de la HPPI, para poner de manifiesto un cuadro característico probablemente infradiagnosticado.

\section{Caso Clínico}

Niña de 11 años de edad, evaluada en el Servicio de Urgencia por lesiones dolorosas en planta de pie derecho de 48 horas de evolución, que impedían la deambulación, con fiebre de hasta $38,2^{\circ} \mathrm{C}$, sin otros síntomas ni signos clínicos cutáneos ni sistémicos asociados.

Como únicos antecedentes de interés, la paciente refería episodios habituales de hiperhidrosis plantar y práctica de baloncesto en los días previos. En la exploración física se observaban nódulos eritematovioláceos, dolorosos a la palpación, en borde lateral externo y zona de antepié de planta de pie derecho (figura 1).

Se realizó una biopsia cutánea de una de las lesiones, que mostró un infiltrado inflamatorio de predominio neutrofílico alrededor de las glándulas sudoríparas ecrinas y abscesos de neutrófilos en las proximidades del ovillo (figura 2). Con estos hallazgos se confirmó la sospecha diagnóstica de HPPI y se inició tratamiento con antiinflamatorios no esteroidales orales y reposo, con resolución paulatina de las lesiones en 7 días, sin presentar secuelas ni recidivas tras 3 años de seguimiento.

\section{Discusión}

La HPPI es una enfermedad propia de niños y adultos jóvenes, caracterizada clínicamente por la presencia de placas y/o nódulos eritematosos o eritematovioláceos, dolorosos, de comienzo brusco, localizados 


\section{Tabla 1. Diagnóstico diferencial de lesiones plantares dolorosas}

\begin{tabular}{|c|c|c|c|c|c|}
\hline & HPPI & HEN & UTP & ENP & SPCP \\
\hline Clínica & $\begin{array}{l}\text { Nódulos dolorosos } \\
\text { palmoplantares de } \\
\text { aparición brusca uni/ } \\
\text { bilaterales +/- fiebre }\end{array}$ & $\begin{array}{l}\text { Erupción febril poli- } \\
\text { morfa en tronco, cara } \\
\text { y extremidades }\end{array}$ & $\begin{array}{l}\text { Maculopápulas plan- } \\
\text { tares eritematosas, } \\
\text { habonosas, recurren- } \\
\text { tes, pruriginosas o } \\
\text { dolorosas }\end{array}$ & $\begin{array}{l}\text { Nódulos subcutáneos } \\
\text { plantares dolorosos, } \\
\text { con hiperpigmenta- } \\
\text { ción postinflamatoria } \\
\text { +/- Fiebre, artralgias y } \\
\text { malestar general }\end{array}$ & $\begin{array}{l}\text { Nódulos plantares eri- } \\
\text { tematosos, dolorosos, } \\
\text { de aparición brusca }\end{array}$ \\
\hline $\begin{array}{l}\text { Antecedentes/ } \\
\text { Asociaciones }\end{array}$ & $\begin{array}{l}\text { Niño/joven sano } \\
\text { +/- Hiperhidrosis, } \\
\text { ejercicio físico }\end{array}$ & $\begin{array}{l}\text { Quimioterápicos } \\
\text { (citarabina...) } \\
\text { Inhibidores de BRAF } \\
\text { G-CSF } \\
\text { Linfoma/leucemia } \\
\text { VIH }\end{array}$ & $\begin{array}{l}\text { Horas después de } \\
\text { ejercicio físico intenso }\end{array}$ & $\begin{array}{l}\text { Precedidos de infec- } \\
\text { ción o fármacos }\end{array}$ & $\begin{array}{l}\text { 6-48 horas después } \\
\text { de baño en una } \\
\text { piscina temperada o } \\
\text { caliente. } \\
\text { En forma de brote co- } \\
\text { munitario en piscinas } \\
\text { publicas }\end{array}$ \\
\hline Patogenia & $\begin{array}{l}\text { Lesión gl. ecrinas } \\
\text { inmaduras por alta } \\
\text { temperatura o facto- } \\
\text { res mecánicos }\end{array}$ & $\begin{array}{l}\text { Efecto citotóxico } \\
\text { directo de la secreción } \\
\text { de la quimioterapia } \\
\text { en las gl. ecrinas o Sd. } \\
\text { paraneoplásico }\end{array}$ & $\begin{array}{l}\text { Urticaria física por } \\
\text { presión }\end{array}$ & $\begin{array}{l}\text { Hipersensibilidad } \\
\text { retardada frente a } \\
\text { variedad de estímulos } \\
\text { antigénicos }\end{array}$ & $\begin{array}{l}\text { Infección por Pseudo- } \\
\text { monas Aeruginosa }\end{array}$ \\
\hline Histología & $\begin{array}{l}\text { Abscesos neutrofílicos } \\
\text { alrededor de ovillo y } \\
\text { conductos secretores } \\
\text { de glándulas ecrinas, } \\
\text { en ocasiones en el } \\
\text { interior del conducto } \\
\text { Sin vasculitis }\end{array}$ & $\begin{array}{l}\text { Infiltrado de neu- } \\
\text { trófilos en porción } \\
\text { secretora de las } \\
\text { glándulas ecrinas, der- } \\
\text { mis reticular y tejido } \\
\text { celular subcutáneo. } \\
\text { Siringometaplasia es- } \\
\text { camosa del conducto } \\
\text { ecrino } \\
\text { No abscesos }\end{array}$ & $\begin{array}{l}\text { Infiltrado inflamatorio } \\
\text { de neutrófilos y eosi- } \\
\text { nófilos, con edema, } \\
\text { en la dermis papilar y } \\
\text { reticular sin afecta- } \\
\text { ción de las glándulas } \\
\text { sudoríparas ecrinas }\end{array}$ & $\begin{array}{l}\text { Paniculitis septal sin } \\
\text { vasculitis }\end{array}$ & $\begin{array}{l}\text { Similar a HPPI, salvo } \\
\text { tinción Gram en la } \\
\text { que se puede apreciar } \\
\text { el microorganismo }\end{array}$ \\
\hline Tratamiento & $\begin{array}{l}\text { Autorresolutivo en < } \\
3 \text { semanas } \\
\text { +/- AINES, reposo, } \\
\text { corticoides }\end{array}$ & $\begin{array}{l}\text { Autorresolutivo en } \\
1-3 \text { semanas } \\
+/- \text { Tratamiento } \\
\text { etiológico }\end{array}$ & $\begin{array}{l}\text { Evitar estímulo desen- } \\
\text { cadenante } \\
\text { Antihistamínicos } \\
\text { orales }\end{array}$ & $\begin{array}{l}\text { Reposo +/- salicilatos } \\
\text { o AINES }\end{array}$ & $\begin{array}{l}\text { Evitar la fuente de } \\
\text { infección } \\
\text { Antimicrobianos y } \\
\text { antisépticos tópicos } \\
\text { +/- antibiótico oral }\end{array}$ \\
\hline
\end{tabular}

HPPI (Hidradenitis palmoplantar ecrina idiopática), HEN (hidradenitis ecrina neutrofílica), UTP (urticaria traumática plantar), ENP (eritema nodoso plantar infantil), SPCP (Síndrome de pie caliente por pseudomonas)

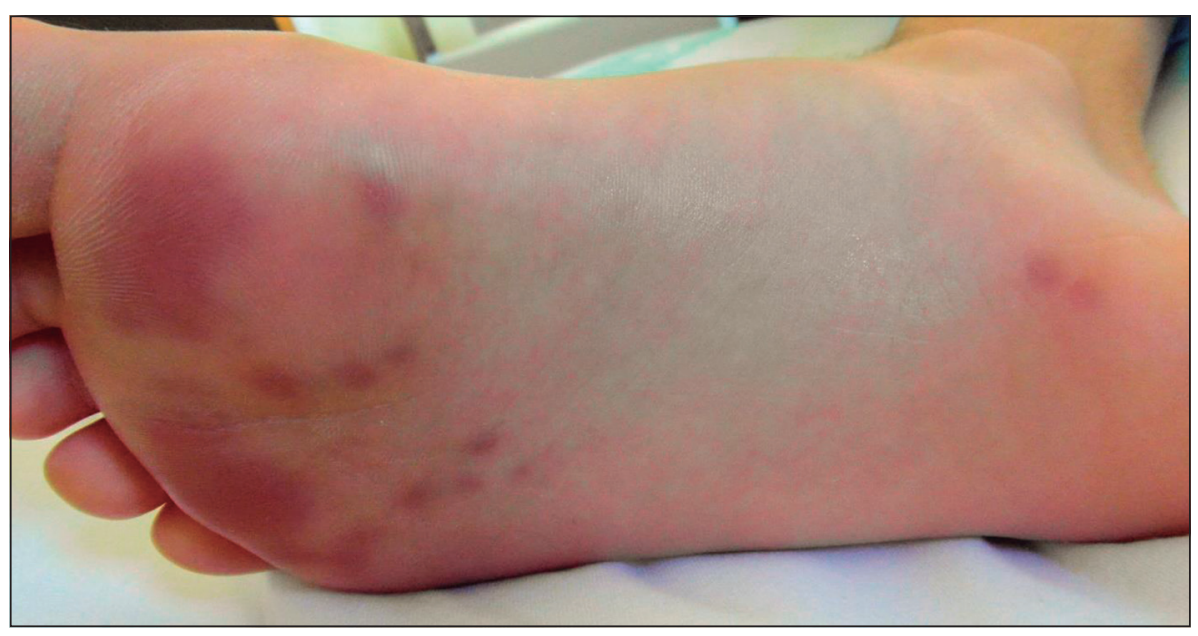

Figura 1. Nódulos eritematovioláceos en zona de antepié y talón. 

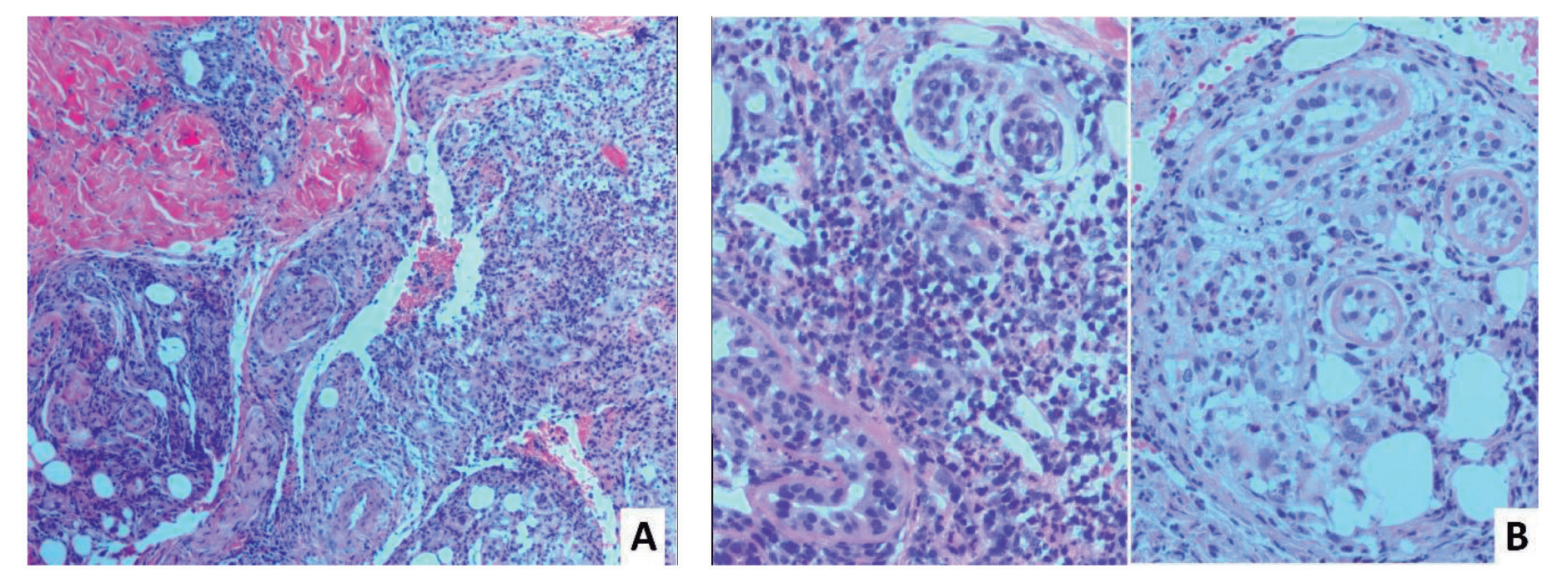

Figura 2. A) HE x20: infiltrado inflamatorio constituido por polimorfonucleares neutrófilos alrededor de las glándulas sudoríparas ecrinas, tanto en la porción del ovillo como en el conducto. B) HE x40: detalle de la infiltración inflamatoria que rodea y ocupa la luz de las glándulas.

en regiones plantares o palmoplantares, de forma uni o bilateral $^{2,4,8}$. Por lo general, este cuadro se presenta en pacientes sanos, sin antecedentes de consumo reciente de medicación ni otras enfermedades subyacentes ${ }^{8}$. En algunos casos se acompaña de fiebre, pero sin otros síntomas generales ${ }^{2,4}$.

Es importante que, ante un paciente en edad pediátrica que consulta por nódulos dolorosos acrales, sepamos reconocer este cuadro, y lo diferenciemos de otros con clínica similar (tabla 1). Los principales diagnósticos diferenciales a tener en cuenta son: la urticaria traumática plantar, caracterizada por brotes recidivantes de maculopápulas eritematosas plantares, pruriginosas o dolorosas, que aparecen horas después de una actividad física intensa, en cuyo estudio histológico aparece un infiltrado inflamatorio en dermis sin afectación de las glándulas sudoríparas ecrinas ${ }^{2,8,9}$; el eritema nodoso plantar infantil, que cursa con nódulos muy dolorosos, generalmente precedidos de infección o consumo de fármacos, que se resuelven de forma más lenta dejando una hiperpigmentación postinflamatoria y en cuya histología encontramos la característica paniculitis septal propia del eritema nodoso ${ }^{2,8,10}$; y el síndrome de pie caliente por pseudomonas, debido a una infección bacteriana por Pseudomonas aeruginosa que presenta una clínica muy similar a la HPPI, con nódulos plantares eritematosos, dolorosos, de aparición brusca, pero que se manifiesta característicamente en forma de brotes comunitarios en niños, a partir de 6 a 48 horas después de haberse bañado en una piscina caliente o temperada contaminada por dicho patógeno ${ }^{11}$. También podrían considerarse en el diagnóstico diferencial otras entidades como el eritema multiforme, la perniosis y algunos cuadros de vasculitis y celulitis ${ }^{2,8}$.

Por último, no debemos confundir la HPPI con la HEN, una erupción febril polimorfa que afecta a tronco, cara y extremidades. Esta entidad fue descrita inicialmente en pacientes con neoplasia hematológica que recibían tratamiento quimioterápico y, más tarde, también ha sido asociada con otros fármacos, neoplasias e infecciones. Ambas presentan una histología similar, pero con características diferenciales como la presencia de siringometaplasia escamosa del conducto ecrino en la HEN, y la presencia de abscesos de neutrófilos próximos a los ovillos glandulares en la HPPI ${ }^{8}$.

En cuanto al tratamiento, se recomienda únicamente alivio sintomático, dado que se trata de un cuadro que se resuelve de forma espontánea en alrededor de 3 semanas sin dejar secuelas. Generalmente éste incluye reposo que puede ir acompañado de antiinflamatorios no esteroideos y corticoides tópicos ${ }^{2,6}$.

\section{Conclusión}

Presentamos un caso de HPPI con un cuadro clínico e histológico característico, y aportamos las claves para hacer un correcto diagnóstico diferencial con otras entidades que cursan con nódulos acrales dolorosos, pero presentan un curso clínico y unas implicaciones terapéuticas distintas, creando una alarma innecesaria, tanto en los pacientes y sus padres, como en los propios médicos.

\section{Responsabilidades Éticas}

Protección de personas y animales: Los autores declaran que los procedimientos seguidos se conformaron a las normas éticas del comité de experimentación hu- 
mana responsable y de acuerdo con la Asociación Médica Mundial y la Declaración de Helsinki.

Confidencialidad de los datos: Los autores declaran que han seguido los protocolos de su centro de trabajo sobre la publicación de datos de pacientes.

Derecho a la privacidad y consentimiento informado: Los autores han obtenido el consentimiento infor- mado de los padres (tutores) de la paciente y/o sujetos referidos en el artículo. Este documento obra en poder del autor de correspondencia

\section{Conflicto de intereses}

Los autores declaran no tener conflicto de intereses.

\section{Referencias}

1. Stahr BJ, Cooper PH, Caputo RV. Idiopathic plantar hidradenitis: a neutrophilic eccrine hidradenitis occurring primarily in children. J Cutan Pathol. 1994;21:289-96.

2. López Blázquez M, Lozano Masdemont B, Mora Capín A, Gómez-Recuero Muñoz L, Campos Domínguez M. Idiopathic palmoplantar hidradenitis: is biopsy always necessary? Acta Pediatr Esp. 2017; 75:e8-e10.

3. Rabinowitz LG, Cintra ML, Hood AF, Esterly NB. Recurrent palmoplantar hidradenitis in children. Arch Dermatol.
1995;131:817-20.

4. Housni Alaoui I, Hocar O, Akhdari N et al. An uncommon cause of delayed walking: idiopathic palmoplantar hidradenitis. Arch Pediatr. 2015; 22:306-8.

5. Naimer SA, Zvulunov A, Ben-Amitai D, Landau M. Plantar hidradenitis in children induced by exposure to wet footwear. Pediatr Emerg Care. 2000;16:182-3.

6. Ben-Amitai D, Hodak E, Landau M, Metzker A, Feinmesser M, David M. Idiopathic palmoplantar eccrine hidradenitis in children. Eur J Pediatr. 2001;160:189-91.

7. Blázquez Sánchez N, Rodrigo Fernández I.
Lesiones plantares recurrentes. Piel. 2007; 22:453-5.

8. González-Hermosa MR, GonzálezGüemes M, González-Pérez R, Catón B, Díaz-Ramón JL, Soloeta R. Hidradenitis palmar idiopática. Actas Dermosifiliogr 2003;94:660-2.

9. Metzker A, Brodsky F. Traumatic plantar urticaria-an unrecognized entity? J Am Acad Dermatol. 1988;18:144-6.

10. Suárez SM, Paller AS. Plantar erythema nodosum: Cases in two children. Arch Dermatol 1993;129:1064-65.

11. Fiorillo L, Zucker M, Sawyer D, Lin AN. The pseudomonas hot-foot syndrome. N Engl J Med. 2001;345:335-8. 